\title{
Valorização da lama do beneficiamento de rochas ornamentais em pavimentos flexíveis
}

\author{
Use of ornamental stone processing sludge in flexible pavements \\ Utilización de lodos de procesamiento de piedra ornamental en pavimentos flexibles
}

Recebido: 05/01/2022 | Revisado: 10/01/2022 | Aceito: 14/01/2022 | Publicado: 16/01/2022

\author{
Renan Tavares Fachin \\ ORCID: https://orcid.org/0000-0003-1893-0110 \\ Universidade do Vale do Rio do Sinos, Brasil \\ E-mail: renan.fachin@hotmail.com \\ Francisco Roger Carneiro Ribeiro \\ ORCID: https://orcid.org/0000-0001-8790-3023 \\ Universidade Federal do Rio Grande do Sul, Brasil \\ E-mail: roger.ribeiro_@hotmail.com \\ Fernanda Pacheco \\ ORCID: https://orcid.org/0000-0003-3455-491X \\ Universidade do Vale do Rio do Sinos, Brasil \\ E-mail: fernandapacheco.fe@gmail.com \\ Feliciane Andrade Brehm \\ ORCID: https://orcid.org/0000-0002-3272-5837 \\ Universidade do Vale do Rio do Sinos, Brasi \\ E-mail: felicianeb@unisinos.br \\ Regina Célia Espinosa Modolo \\ ORCID: https://orcid.org/0000-0001-7088-2502 \\ Universidade do Vale do Rio do Sinos, Brasil \\ E-mail: reginaem@unisinos.br
}

\begin{abstract}
Resumo
O objetivo desse estudo é avaliar a incorporação de lama do beneficiamento de rochas ornamentais como material de enchimento, através de substituições parciais de materiais convencionais, em concreto betuminoso usinado à quente. Foram investigadas as características físico-químicas do resíduo e demais agregados que compõem a mistura asfáltica, seguido do desenvolvimento de formulações para execução do teste de resistência mecânica pelo método Marshall. Os resultados evidenciam que a substituição de materiais naturais por resíduos de rochas ornamentais melhora ligeiramente as propriedades físicas e mecânicas das misturas betuminosas. Concluiu-se, portanto, que a lama do beneficiamento de rochas ornamentais apresenta potencial de utilização e pode ser um material promissor a ser utilizado pela indústria da pavimentação, evitando assim a extração mineral de recursos naturais não renováveis.

Palavras-chave: Resíduo sólido industrial; Lama do beneficiamento de rochas ornamentais; Fíler; Concreto betuminoso usinado à quente.

Abstract

The objective of this study is to evaluate the incorporation of sludge from the processing of ornamental rocks, as a filling material, through partial replacements of conventional materials, in hot machined bituminous concrete. The physical-chemical characteristics of the residue and other aggregates that make up the asphalt mixture were investigated, followed by the development of formulations to perform the mechanical resistance test using the Marshall method. The results show that the replacement of natural materials by waste ornamental rocks slightly improves the physical and mechanical properties of bituminous mixtures. It was therefore concluded that the slurry from ornamental stone processing has potential for use and can be a promising material to be used by the paving industry. Added to this is the fact that consumption of non-renewable natural resources is reduced, since fewer conventional raw materials can be replaced by this waste, thus avoiding mineral extraction.
\end{abstract}

Keywords: Solid industrial waste; Sludge from the processing of ornamental rocks; Filler; Hot machined bituminous concrete.

\section{Resumen}

El objetivo de este estudio es evaluar la incorporación de barro en el beneficio de las rocas ornamentales como material de recubrimiento, a través de sustituciones parciales de materiales convencionales, en el hormigón betuminoso utilizado en el quente. Se han investigado las características físico-químicas del material y de los agregados que componen la mezcla asfáltica, seguido del desarrollo de fórmulas para la ejecución de la prueba de 
resistencia mecánica por el método Marshall. Los resultados muestran que la sustitución de los materiales naturales por roca residual mejora ligeramente las propiedades físicas y mecánicas de las mezclas bituminosas. Se concluye, por tanto, que la lama del beneficio de las rocas ornamentales presenta un potencial de utilización y puede ser un material prometedor para ser utilizado por la industria de la pavimentación, evitando así la extracción mineral de recursos naturales no renovables.

Palabras clave: Residuos industriales sólidos; Lodos procedentes del procesamiento de rocas ornamentales; Rellenos; Hormigón bituminoso mecanizado en caliente.

\section{Introduçãa}

A malha rodoviária mundial consiste em mais de 36 milhões de quilômetros dentre estradas pavimentadas e não pavimentadas (CIA, 2017). Essas estradas pavimentadas são compostas por camadas de diferentes materiais granulares e ligantes asfálticos, cuja construção e manutenção depende da disponibilidade e extração frequente de recursos naturais nãorenováveis, o que viola assim o desenvolvimento de uma infraestrutura sustentável. De acordo com Chen e Xu (2010), o esqueleto estrutural dos pavimentos é constituído por agregados e pelo cimento asfáltico de petróleo (CAP) usado como ligante da mistura mineral. Esse último é um material de alta viscosidade produzido por meio de resíduos da destilação do petróleo e possui característica de um termoplástico.

Os pavimentos asfálticos retratam um processo construtivo importante para a vida cotidiana, em virtude do crescente e contínuo volume de tráfego, nos quais há a necessidade de misturas e aglutinantes com propriedades aprimoradas para garantir a durabilidade e o desempenho funcional da infraestrutura de transportes. Nessa perspectiva, o desenvolvimento de tecnologias com eficiência energética e ambientalmente corretas apresenta-se como um mercado promissor (Wang et al., 2018; Xiong et al., 2019).

De acordo com Zhang et al. (2016), devido ao rápido desenvolvimento da indústria, elevadas quantidades de resíduos são produzidas e por vezes descartadas de maneira inadequada, apesar de muitos possuírem características que possibilitam a sua reciclagem corroborando um sistema produtivo mais "limpo".

A lama do beneficiamento de rochas ornamentais (LBRO) é oriunda do corte e do polimento de rochas ornamentais (mármore, granito, calcário, basalto, dentre outros), apresentando-se como um material muito fino que tem sido testado como carga na produção de materiais de construção. O mármore, por exemplo, possui minerais de calcita e dolomita em sua composição e por serem insolúveis em água, formam melhores ligações com o betume, o que resulta em misturas mais resistentes à umidade. O granito, por sua vez, apesar de deter uma menor quantidade de calcita, também não apresenta influência negativa à umidade nas misturas (Chandra \& Choudhary, 2013), pois é rico em quartzo.

No entanto, esses resíduos possuem propriedades distintas a depender da qualidade do mineral e do processo de tratamento na indústria geradora, uma vez que a interação com o betume pode afetar significativamente o desempenho e a durabilidade do material asfáltico (Modarres et al., 2015, Li et al., 2018), assim como o custo final do produto. O desempenho insatisfatório dos pavimentos encontra-se atrelado ao rápido crescimento das cargas de tráfego e ao peso de eixos dos veículos, tendo em vista a produção das misturas asfálticas com polímeros e/ou cargas minerais de baixa qualidade sem um equilíbrio entre os constituintes (Crispino et al., 2013).

Apesar de serem limitadas, o teor de fíler pode satisfazer algumas propriedades, sejam as: especificações gradativas de agregados, resistência ao surgimento de trincas, resistência à deformações em altas e baixas temperaturas, volumetria, sensibilidade à umidade e desempenho térmico (Aljassar \& Metwali; Ali, 2004, Whang et al., 2011, Zulkati et al., 2012, Chandra \& Choudhary, 2013, Matos et al., 2014, Pasandín \& Pérez, 2016). Assim sendo, e considerando a necessidade de uma produção com menor impacto ambiental, novas misturas asfálticas com o uso de resíduos sólidos podem contribuir ainda para o ganho de benefícios econômicos e ambientais tangíveis para a indústria do asfalto, como o custo do produto final e a redução das emissões de dióxido de carbono pelo menor consumo energético (Behl et al., 2013, Rahman et al., 2015). 
Diante do exposto, há duas maneiras em que os resíduos sólidos industriais podem ser incorporados: por via seca, onde são misturados com os agregados minerais a quente, antes da inserção do ligante asfáltico ou por via úmida, onde os resíduos são inseridos na mistura de agregados e ligante asfáltico. Contudo, estudos apontam que o principal uso desse tipo de resíduo é por meio de substituições dos agregados, com os quais representam cerca de $90 \%$ da mistura total, em massa (Hansan et al., 2019).

Portanto, o presente artigo propõe avaliar a potencial utilização da lama do beneficiamento de rochas ornamentais, como carga mineral, por meio de substituições parciais de materiais naturais na produção de concreto betuminoso usinado à quente (CBUQ) para pavimentos flexíveis.

\section{Materiais e Métodos}

Para o estudo laboratorial, foram utilizados agregados graníticos de diferentes dimensões: Brita 1 (9,5 a 19 mm), Brita 0 (4,8 a 9,5 mm) e pó de pedra $(<4,8 \mathrm{~mm})$ provenientes de britagem fornecidos pela empresa Eldorado Mineração e a lama do beneficiamento de rochas ornamentais, proveniente da empresa Ranzan Pedras. O CAP utilizado como ligante foi o 50/70. A Figura 1 apresenta um fluxograma com os ensaios utilizados para a realização dessa pesquisa.

Figura 1. Ensaios físico-químicos dos materiais.

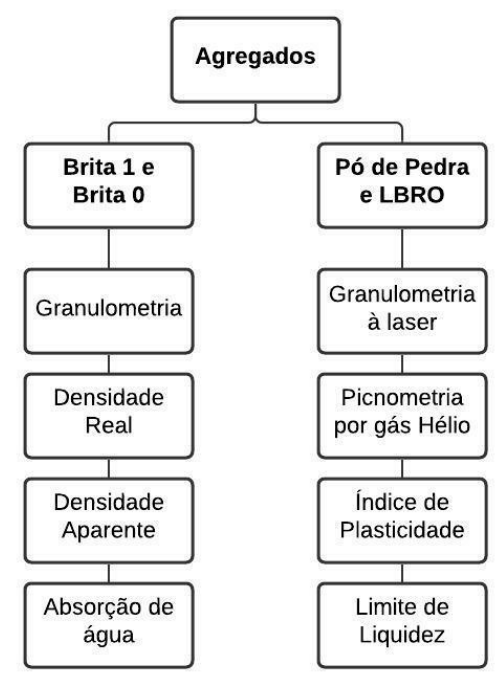

Fonte: Autores.

Com base nos resultados de caracterização dos agregados de acordo com as normas brasileiras NBR 7180 (ABNT, 2016) e NBR 6459 (ABNT, 2017), procedeu-se o desenvolvimento das formulações de misturas asfálticas a partir da Dosagem Marshall em escala laboratorial, na qual adotou-se uma constituição equivalente à faixa $C$ do DNIT 031:2006-ES, que confere um consumo de CAP predominante de 4,5\% em massa total da mistura. Em seguida, definiram-se os teores de substituição de pó de pedra por LBRO em 0\%, 2\% e 4\% para compor o concreto asfáltico de acordo com a composição e distribuição granulométrica das misturas apresentadas na Tabela 1 e na Figura 2, respectivamente. 
Research, Society and Development, v. 11, n. 1, e58711125404, 2022

(CC BY 4.0) | ISSN 2525-3409 | DOI: http://dx.doi.org/10.33448/rsd-v11i1.25404

Tabela 1 - Composição granulométrica das misturas asfálticas sem adição de CAP.

\begin{tabular}{c|c|c|c|c}
\hline Materiais & Brita 1 (\%) & Brita 0 (\%) & Pó de Pedra (\%) & LBRO (\%) \\
\hline Misturas & & & & \\
\hline REF. & 19 & 13 & 68 & 0 \\
\hline A & 19 & 13 & 66 & 2 \\
\hline B & 19 & 13 & 64 & 4 \\
\hline
\end{tabular}

Fonte: Autores.

Figura 2. Distribuição granulométrica das misturas asfálticas sem adição de CAP.
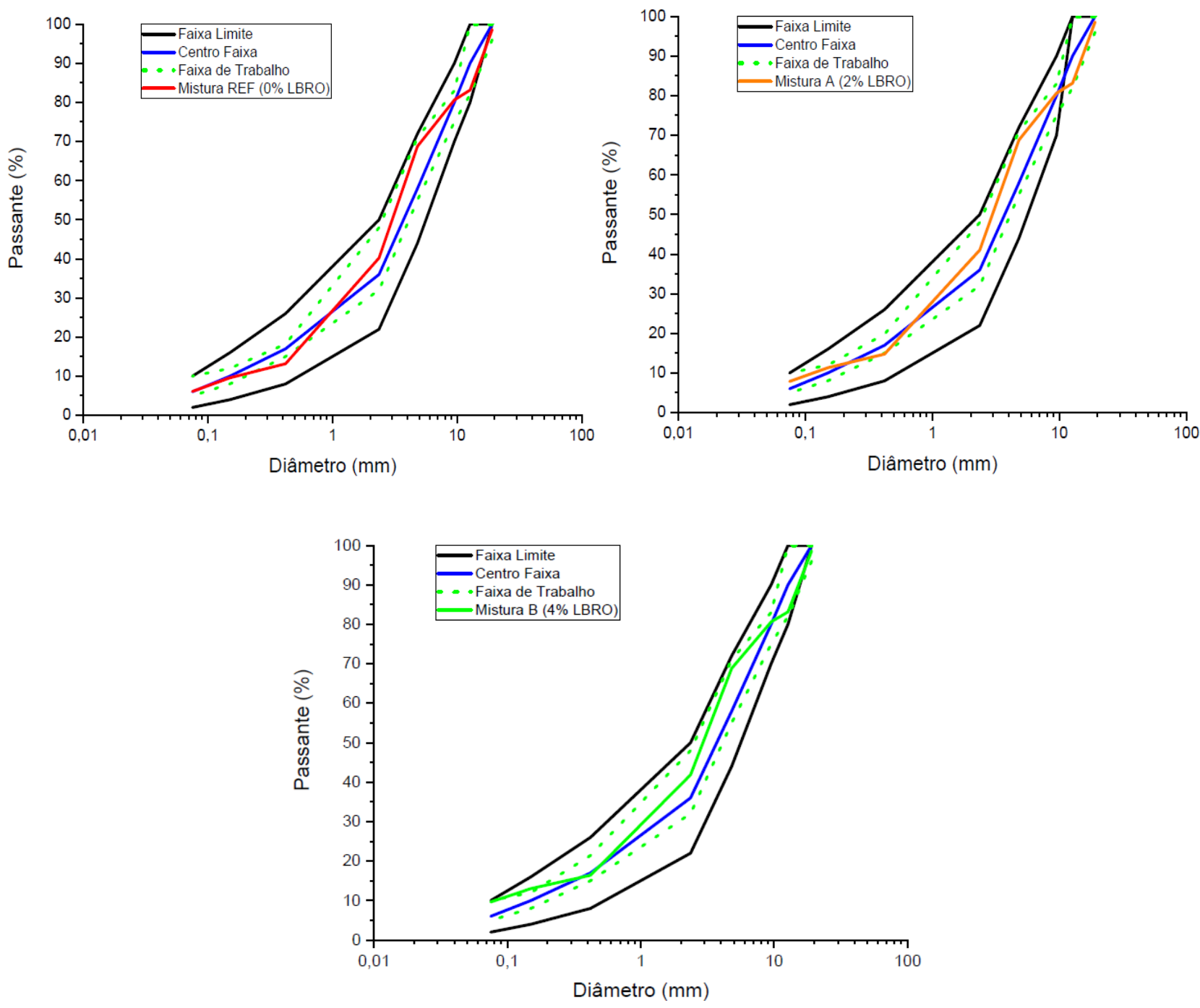

Fonte: Autores.

Posteriormente, moldaram-se cinco corpos de provas cilíndricos de dimensões (100mm x 60mm) para cada composição definida, em que cada corpo de prova possuía uma massa total (agregados + CAP) de aproximadamente 1200 gramas, de acordo com DNIT 178:2018. Por fim, foram aferidas as propriedades físicas: porcentagem de vazios da mistura, percentual de vazios do agregado mineral e relação betume-vazios conforme normativa DNIT 031:2006 - ES e as propriedades mecânicas: estabilidade e fluência, ambas conforme a normativa DNER-ME 043:1995.

\section{Resultados e Discussão}

A Tabela 2 apresenta as características dos materiais utilizados para a produção das misturas asfálticas. 
Tabela 2 - Caracterização físico-química dos agregados.

\begin{tabular}{l|c|c|c|c}
\hline \multicolumn{1}{c|}{ Parâmetros / Materiais } & Brita 1 & Brita 0 & Pó de Pedra & LBRO \\
\hline Diâmetro Máximo Característico $(\mathrm{mm})$ & 19 & 9,5 & Figura 3 & Figura 3 \\
\hline Densidade Real $\left(\mathrm{kg} / \mathrm{m}^{3}\right)$ & 2900 & 2870 & NA & NA \\
\hline Densidade Aparente $\left(\mathrm{kg} / \mathrm{m}^{3}\right)$ & 2780 & 2710 & NA & NA \\
\hline Absorção de Água $(\%)$ & 2,82 & 2,76 & NA & NA \\
\hline Picnometria a gás Hélio $\left(\mathrm{kg} / \mathrm{m}^{3}\right)$ & NA & NA & 2796 & 2670 \\
\hline Índice de Plasticidade & NA & NA & Não plástico & Não plástico \\
\hline Limite de Liquidez $(\%)$ & NA & NA & 19,50 & 23,40 \\
\hline
\end{tabular}

*NA - Não se aplica. Fonte: Autores.

Figura 3. Granulometria à laser do pó de pedra e da LBRO.

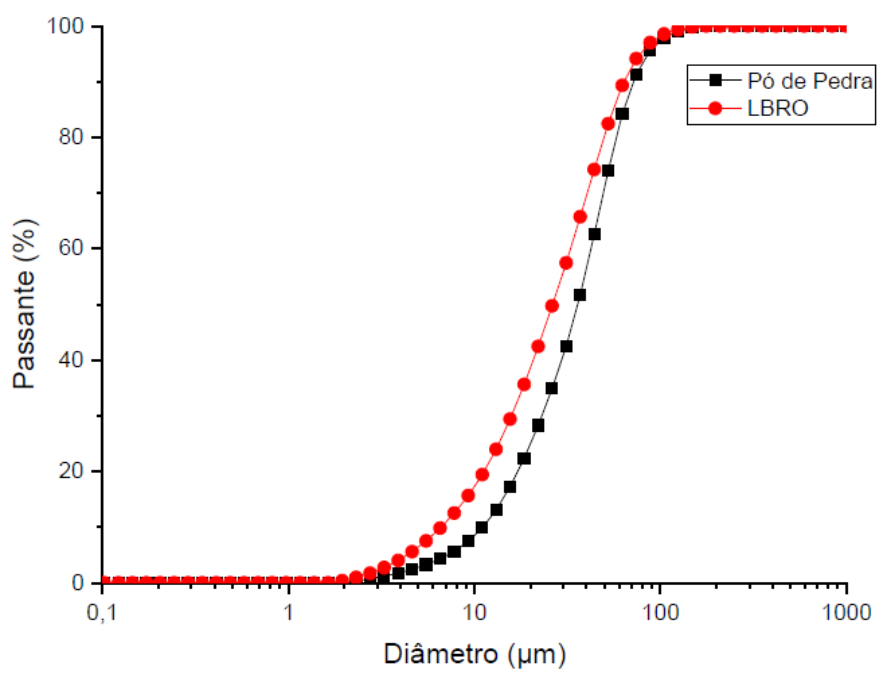

Fonte: Autores.

Os resultados apresentados evidenciam o caráter de agregados graúdos das britas e de agregados miúdos para o pó de pedra e LBRO, uma vez que o ensaio de distribuição granulométrica é fundamental na composição e no desempenho das misturas asfálticas, especificamente estabilidade, trabalhabilidade, rigidez, fadiga, durabilidade e resistência a danos por umidade (Roberts et al., 1996). Por esse ensaio, foi possível confirmar a utilização da LBRO como material de enchimento, tendo em vista que para a Asphalt Institute (2001) é necessário que a maior parte da fração de agregado fino de um certo material passe pela peneira de $75 \mu \mathrm{m}$. Agregados de espessuras e comprimentos elevados são indesejáveis, devido às suas altas áreas superficiais e perdas de trabalhabilidade da mistura asfáltica (ASTM D4791, 2005).

Em relação as densidades, obteve-se dados próximos aos encontrados por Barra et al. (2014). Para West e James (2006) os materiais de carga com densidade específica mais baixa ocupam maiores volumes na mistura e podem deixar menos vazios para acomodar o betume, o que pode promover a redução do consumo de ligante pela mistura.

O ensaio de absorção de água visa garantir o armazenamento adequado dos materiais por meio da capacidade destes de captarem água/umidade. Diante disso, os resultados retratam valores menores que 5\% da massa total para todos os constituintes da mistura asfáltica, confirmando os baixos índices dos agregados absorverem água e/ou umidade em condições atmosféricas e de acordo com os estudos de Karasahin e Terzi (2007), Chandra e Choudhary (2013).

Por fim, no que concerne ao índice de plasticidade e limite de liquidez do pó de pedra e da LBRO, constatou-se que ambos foram definidos como não plásticos, corroborando com investigações de Amaral et al. (2019) e semelhança de teores de umidade ótima conforme apresentado na Figura 4. 
Figura 4. Limites de liquidez do pó de pedra e da LBRO.

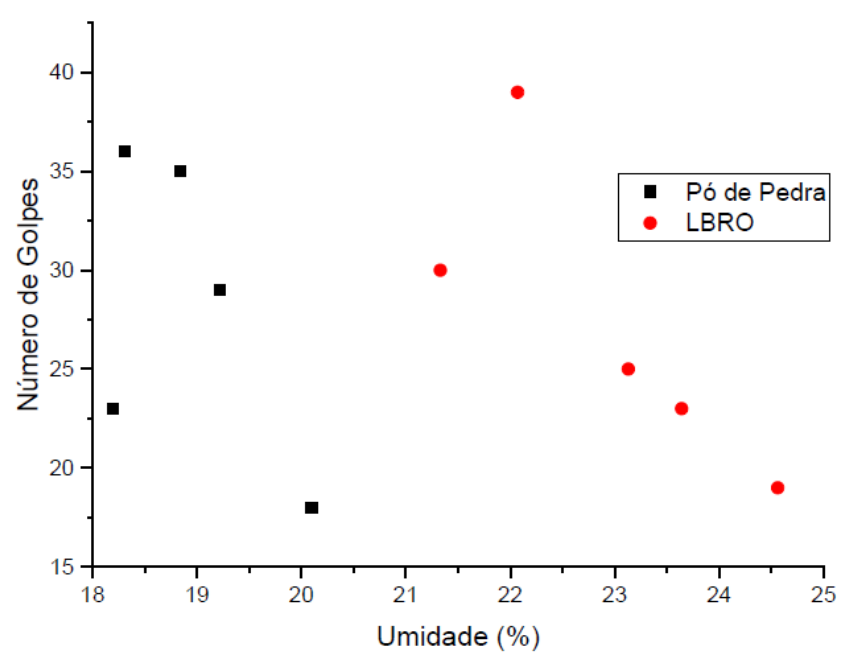

Fonte: Autores.

Os resultados das propriedades físicas das amostras de misturas asfálticas com a utilização de LBRO em suas composições são apresentadas na Figura 5. Nos ensaios de resistência mecânica, nos parâmetros de fluência e estabilidade, optou-se por excluir os resultados de corpos de prova com maior discrepância em cada mistura, bem como fatores de correção (em função da altura do corpo de prova) foram aplicados seguindo o DNER-ME 043:1995, conforme resultados na Figura 6. O desvio padrão das formulações no ensaio de estabilidade não ultrapassou 3,50\% e no ensaio de fluência não ultrapassou $0,85 \%$.

Figura 5. Ensaios físicos das misturas asfálticas.

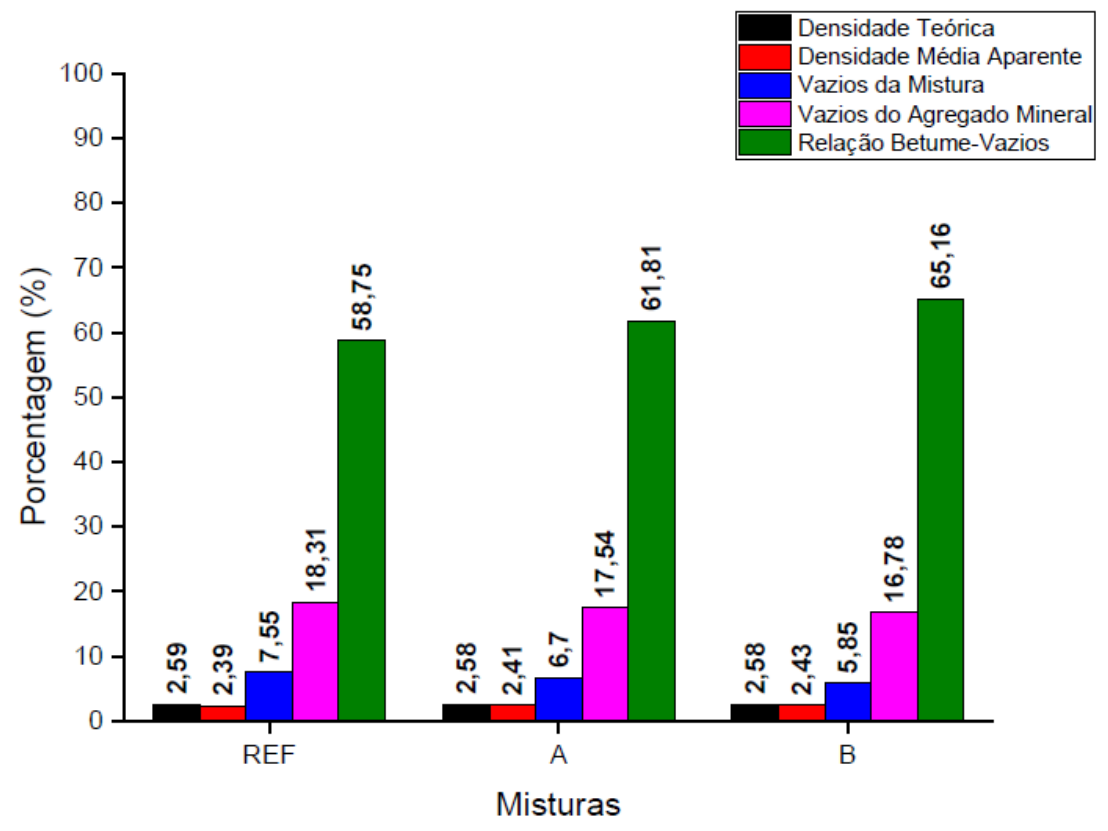

Fonte: Autores. 
Figura 6. Ensaios mecânicos das misturas asfálticas.
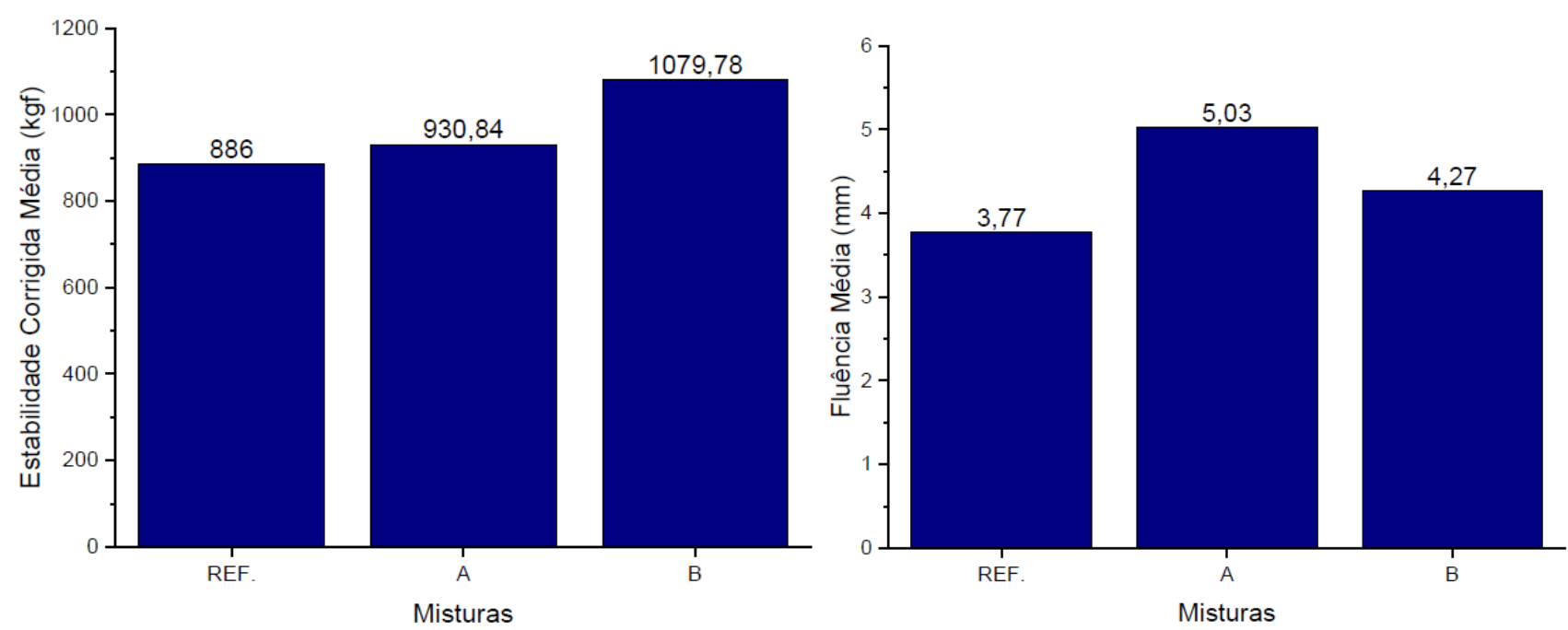

Fonte: Autores.

Modolo et al. (2010) entendem por estabilidade, o valor da força vertical máxima aplicada no corpo de prova e por fluência, o valor da deformação vertical sofrida pela amostra imediatamente antes da ruptura. Na Figura 6 é possível verificar que a estabilidade corrigida média das misturas A e B apresentaram valores superiores em relação a mistura REF. em 5\% e $21,87 \%$, respectivamente. Esse fato pode ser explicado pela maior densificação das misturas asfálticas com a utilização dos resíduos de rochas ornamentais, com a qual pode ter ocorrido um maior empacotamento das partículas com o betume e devido ao menor percentual de vazios médio conforme Figura 5.

Karasahin e Terzi (2007) afirmam que, a depender da quantidade de carga, esse material preenche o vazio dentro da amostra e aumenta a sua estabilidade. Estudos de Wei et al. (2020) complementam o citado, indicando a necessidade de as misturas asfálticas conterem resíduos de rochas para uma maior ligação agregado-betume de maneira mais coesiva para resistir às forças de deformação.

A lógica supracitada possui relação direta com o ensaio de fluência das misturas, uma vez que para diferentes proporções de carga/betume os resultados de fluência aumentam (Karasahin; Terzi, 2007). Todavia, a mistura B apresentou um valor de 15,10\% inferior quando comparado com o resultado obtido pela mistura A, que foi de 13,26\% maior, em relação a mistura REF. Apesar disso, a mistura B se apresentou com o maior valor de estabilidade corrigida média.

\section{Considerações Finais}

Investigou-se a utilização da lama do beneficiamento de rochas ornamentais na produção de misturas asfálticas usinadas à quente, cujos resultados obtidos apresentam-se satisfatórios em relação aos parâmetros analisados. Foi possível constatar que, à medida que o teor de substituição aumenta, os valores de estabilidade e de fluência tendem a aumentar. Porém, na mistura com $4 \%$ de incorporação de LBRO, os resultados foram inversamente proporcionais. Ademais, verificou-se que as características físicas e químicas das cargas são responsáveis por fornecer desempenhos satisfatórios em comparação com a mistura referencial.

O estudo ora apresentado demonstra que a inserção de LBRO, além de contribuir com a preservação do meio ambiente, ao mitigar os impactos ambientais da disposição inadequada desse resíduo e reduzir a extração de recurso natural não renovável (pó de pedra) na produção de mistura asfáltica, agrega valor ao produto final ao produzir um mástique de melhor 
qualidade e pode promover a redução dos teores de CAP, de maneira a beneficiar a indústria de rochas ornamentais e o setor de pavimentação.

Como sugestão para trabalhos futuros, recomenda-se construir trechos de estradas experimentais com as misturas estudadas para avaliar os desempenhos sob ações de tráfegos leves e/ou pesados, bem como quando expostas às diferentes condições metereológicas.

\section{Agradecimentos}

Os autores agradecem ao Conselho Nacional de Desenvolvimento Científico e Tecnológico (CNPq) pela Bolsa de Produtividade em Pesquisa - Nível 2 da autora Regina Célia Espinosa Modolo, pela Bolsa de Produtividade em Desenvolvimento Tecnológico e Extensão Inovadora - Nível 2 da autora Feliciane Andrade Brehm e à Coordenação de Aperfeiçoamento de Pessoal de Nível Superior (CAPES) pela Bolsa CAPES/PROSUC do autor Francisco Roger Carneiro Ribeiro.

\section{Referências}

Aljassar, A. H., Metwalli, S., \& Ali, M. A. (2004). Effect of Filler Types on Marshall Stability and Retained Strength of Asphalt Concrete. International Journal of Pavement Engineering, 5, 47-51.

Amaral, L.F., Carvalho, R. P. R. G., Silva, B. M., Delaqua, D. C. G., Monteiro, S. N., \& Vieira, C. M. F. (2019). Development of ceramic paver with ornamental rock waste. Journal of Materials Research and Technology, 8, 599-608.

American Society for Testing and Materials. (2005). ASTM D4791: Standard Test Method for Flat Particles, Elongated Particles, or Flat and Elongated Particles in Coarse Aggregate.

Arab, P. B., Araujo, T. P., \& Pejon, O. J. (2015). Identification of clay minerals in mixtures subjected to differential thermal and thermogravimetry analyses and methylene blue adsorption tests. Applied Clay Science, 114, 133-140.

Asphalt Institute. (2001). HMA Construction. Manual Series No. 22.

Associação Brasileira de Normas Técnicas. (2016). NBR 7180: Solo - Determinação do limite de plasticidade.

Associação Brasileira de Normas Técnicas. (2017). NBR 6459: Solo - Determinação do limite de liquidez.

Barra, B., Momm, L., Guerrero, Y., \& Bernucci, L. (2014). Characterization of granite and limestone powders for use as fillers in bituminous mastics dosage. Anais da Academia Brasileira de Ciências, 86, 995-1002.

Behl, A., Kumar, G., Sharma, G., \& Jain, P. K. (2013). Evaluation of field performance of warm-mix asphalt pavements in India. Procedia - Social and Behavioral Sciences, 104, 158-167.

Chandra, S., \& Choudhary, R. (2013). Performance characteristics of bituminous concrete with industrial wastes as filler. Journal of Materials in Civil Engineering, 25, 1666-1673.

Chen, H., \& Xu, Q. (2010). Experimental study of fibers in stabilizing and reinforcing asphalt binder. Fuel, 89, 1616-1622.

Cia. (2017). Available From: https://www.cia.gov/library/publications/the-worldfactbook/ rankorder/2085rank.html.

Crispino, M., Mariani, E., \& Toraldo, E. (2013). Assessment of fiber-reinforced bituminous mixtures compaction temperatures through mastics viscosity tests Construction and Building Materials, 38, 1031-1039.

Departamento Nacional de Estradas de Rodagem. (1995). DNER-ME 043: Mistura betuminosas a quente - Ensaio Marshall.

Departamento Nacional de Infraestrutura de Transportes. (2006). DNIT ES 031: Pavimentos flexíveis - Concreto asfáltico - Especificações de serviço.

Hasan, M.R.M., Chew, J., Jamshidi, A., Yang, X., \& Hamzah, M. O. (2019). Review of sustainability, pretreatment, and engineering considerations of asphalt modifiers from the industrial solid wastes. Journal of Traffic and Transportation Engineering, 6, 209-244.

Karasahin, M., \& Terzi, S. (2007). Evaluation of marble waste dust in the mixture of asphaltic concrete. Construction and Building Materials, 21, 616-620.

Li, Q., Yanjun, Q., Rahman, A., \& Ding, H. (2018). Application of steel slag powder to enhance the low-temperature fracture properties of asphalt mastic and its corresponding mechanism. Journal of Cleaner Production, 184, 21-31.

Matos, P., Micaelo, R., Duarte, C., \& Quaresma, L. (2014). Influence of bitumen and filler on the selection of appropriate mixing and compaction temperatures. International Journal of Pavement Research and Technology, 7, 237-246. 
Research, Society and Development, v. 11, n. 1, e58711125404, 2022

(CC BY 4.0) | ISSN 2525-3409 | DOI: http://dx.doi.org/10.33448/rsd-v11i1.25404

Modarres, A., Rahmanzadeh, M., \& Ayar, R. (2015). Effect of coal waste powder in hot mix asphalt compared to conventional fillers: mix mechanical properties and environmental impacts. Journal of Cleaner Production, 91, 262-268.

Modolo, R., Benta, A., Ferreira, V. M., \& Machado, L. M. (2010). Pulp and paper plant wastes valorisation in bituminous mixes. Waste Management, 30, 685696.

Pasandín, A. R., Pérez, I., Ramírez, A., \& Cano, M. M. (2016). Moisture damage resistance of hot-mix asphalt made with paper industry wastes as filler. Journal of Cleaner Production, 112, 853-862.

Rahman, Md. A., Imteaz, M. A., Arulrajah, A., Piratheepan, J., \& Disfani, M. M. (2015). Recycled construction and demolition materials in permeable pavement systems: geotechnical and hydraulic characteristics. Journal of Cleaner Production, 90, 193-194.

Roberts, F. L., Brown, E. R., Kandhal, P. S., Lee, D., Kennedy, T. W., \& Kim, Y. R. (1996). Hot mix asphalt materials, mixture design and construction.

Wang, H., Al-Qadi, I. L., Faheem, A. F., Bahia, H. U., Yang, S., \& Reinke, G. H. (2011). Effect of mineral filler characteristics on asphalt mastic and mixture rutting potential. Transportation Research Record, 2208, 33-39.

Wang, H., Liu, X., Apostolidis, P., \& Scarpas, T. (2018). Review of warm mix rubberized asphalt concrete: Towards a sustainable paving technology. Journal of Cleaner Production, 177, 302-314.

Wei, C. J., Poovaneshvaran, S., Hasan, M. R. M., Hamzah, M. O., Valentin, J., \& Sani, A. (2020). Microscopic analysis and mechanical properties of recycled paper mill sludge modified asphalt mixture using granite and limestone aggregates. Construction and Building Materials, 243.

West, R. C., \& James, R. S. (2006). Evaluation of a lime kiln dust as a mineral filler for stone matrix asphalt. Transportation Research Board, 750, 1-18.

Xiong, R., Chu, C., Qiao, N., Wang, L., Yang, F., Sheng, Y., Guan, B., Niu, D., Geng, J., \& Chen, H. (2019). Performance evaluation of asphalt mixture exposed to dynamic water and chlorine salt erosion. Construction and Building Materials, 201, 121-126.

Zhang, M., Wang, Y., Song, Y., Zhang, T., \& Wang, J. (2016). Manifest system for management of non-hazardous industrial solid wastes: results from a Tianjin industrial park. Journal of Cleaner Production, 133, 252-261.

Zulkati, A., Diew, W. Y., \& Delai, D. S. (2012). Effects of fillers on properties of asphalt-concrete mixture. Journal of Transportation Engineering, 138, 902910. 